\title{
Naprapathy attenuates neuropathic pain after brachial plexus injury
}

\author{
Bin Xiao" ${ }^{1 \#}$, Anqi Ma ${ }^{1 \#}$, Zhengyu Li ${ }^{1}$, Shenyu Zhang ${ }^{2}$, Xiaojun $\mathrm{Xu}^{2}$, Junming Zhou ${ }^{2}$, Weijiang $\mathrm{Li}^{1}$, \\ Jing Zhang ${ }^{1}$, Fei Yao ${ }^{1}$
}

${ }^{1}$ College of Acupuncture and Tuina, Shanghai University of TCM, Shanghai 201203, China; ${ }^{2}$ Department of Hand Surgery, Huashan Hospital, Fudan University, Key Laboratory of Hand Reconstruction, Ministry of Health, Shanghai Key Laboratory of Peripheral Nerve and Microsurgery, Shanghai 200040, China

Contributions: (I) Conception and design: B Xiao, A Ma, Z Li, J Zhang; (II) Administrative support: All authors; (III) Provision of study materials or patients: All authors; (IV) Collection and assembly of data: B Xiao, A Ma, Z Li, S Zhang; (V) Data analysis and interpretation: B Xiao, A Ma; (VI) Manuscript writing: All authors; (VII) Final approval of manuscript: All authors.

"These authors contributed equally to the work.

Correspondence to: Zhengyu Li. College of Acupuncture and Tuina, Shanghai University of TCM, Shanghai 201203, China. Email: lzy1855@aliyun.com; Junming Zhou. Department of Hand Surgery, Huashan Hospital, Fudan University, Key Laboratory of Hand Reconstruction, Ministry of Health, Shanghai Key Laboratory of Peripheral Nerve and Microsurgery, Shanghai 200040, China. Email: zjm2585@163.com.

Background: This study was to explore the potential mechanism of naprapathy in treating neuropathic pain (NP) after brachial plexus injury (BPI).

Methods: Totally 72 rats were randomly divided into normal group, model control group, and naprapathy group ( $\mathrm{n}=24$ per group). A right upper-limb chronic NP model was established, and naprapathy was administered at C5-T1 Jiaji Points on 4th day after modeling. Naprapathy was performed for 15 min once daily with a frequency of 60 per minute. The treatment was applied for altogether 28 days. Cold pain threshold and mechanical pain threshold were measured 1 day before modeling, 3 days after modeling, and 7,14, 21 and 28 days after naprapathy; 7, 14, 21 and 28 days after naprapathy, rats were killed and the $\beta$-endorphin expression and $\gamma$-aminobutyric acid (GABA) content were detected in the thalamus.

Results: After 14-day treatment, there was significant difference of mechanical pain threshold between the naprapathy group and the normal group $(\mathrm{P}<0.05)$; after treatment for 21 and 28 days, there was no significant difference between the naprapathy group and the normal group $(\mathrm{P}>0.05)$; after 28-day naprapathy, there was significant difference of $\beta$-EP expression between the normal group and the naprapathy group $(\mathrm{P}<0.05)$, while the difference between model control group and naprapathy group was statistically significant $(\mathrm{P}<0.05)$. After 14-day treatment, there was significant difference of GABA content between the model control group and the naprapathy group $(\mathrm{P}<0.05)$. After 28-day treatment, significant difference was also found between the model control group and the naprapathy group $(\mathrm{P}<0.05)$.

Conclusions: After naprapathy, chronic NP is attenuated in rats with BPI, which might be ascribed to the upregulation of $\beta$-endorphin and GABA.

Keywords: Nerve regeneration; peripheral nerve injury; brachial plexus injury (BPI); neuropathic pain (NP); naprapathy; $\beta$-endorphin; $\gamma$-aminobutyric acid (GABA)

Submitted Sep 02, 2019. Accepted for publication Feb 14, 2020.

doi: 10.21037/apm.2020.04.16

View this article at: http://dx.doi.org/10.21037/apm.2020.04.16 


\section{Introduction}

Brachial plexus injury (BPI) is one of the most serious peripheral nerve injuries and often causes severe dysfunction of the upper limbs or even life-long disability $(1,2)$. Treatment of BPI has been one of the medical challenges. Although the examination of BPI has been greatly improved in recent years, which increases the diagnostic rate and the understanding of this disease and, at the same time, improves its surgical treatment, no effective solution is available to the neuropathic pain (NP) after nerve injury. Brachial plexus avulsion can cause immediate or delayed pain, which is characterized by squeezing, crushing and burning-like colica, allodynia, spontaneous pain and hyperalgesia, or the combination of these types of pain. Furthermore, the post-injury pain may develop into a chronic, refractory NP, which may even remain after surgery (3). Thus, most patients with BPI usually suffer from long-term chronic pain, which may even significantly affect the disease prognosis (4).

Naprapathy is an external treatment method in the Traditional Chinese Medicine (TCM), and its efficacy in relieving pain has been proved in clinical practice. The ancient Chinese literature "Inner Canon of the Yellow Emperor" clearly points out that naprapathy has the capabilities of dredging meridians and dredging collateral as well as promoting flow of Qi and blood circulation and activating meridians to stop pain. In most cases, the pain is caused by "Qi-blood obstruction" and/or "Qi-blood insufficiency" based on the TCM theory. In the present study, we investigated the effects of naprapathy on the NP secondary to BPI in a rat model, and the $\beta$-endorphin expression and GABA content were detected in the brain, aiming to explore the potential mechanism underlying the therapeutic effects of naprapathy.

\section{Methods}

\section{Animals}

Seventy-two specific pathogen free male Sprague-Dawley rats aged 6-8 weeks (adult) and weighing 180-200 g were purchased from Shanghai Xipuer-Bikai Experimental Animal Co., Ltd. [license number: SCXK (Shanghai) 20130016], and animals were housed in the Experimental Animal Center of Shanghai University of Traditional Chinese Medicine [license number: SYXK (Shanghai) 2014-0008].

Animals were housed in an environment at $20-24{ }^{\circ} \mathrm{C}$ with a humidity of $60-70 \%$ and $12 \mathrm{~h} / 12 \mathrm{~h}$ light/dark cycle (7:00-
19:00). Rats were given ad libitum access to water and food. They were allowed to accommodate to the environment for one week before study.

\section{Instrument}

Electronic Von Frey pain detector (23931, IITC; USA) and Hot and cold plate pain detector (YLS-21A; Jinan Yiyan Technology Co., Ltd.) were used in this study.

\section{Reagent}

The Rabbit Anti-GABABR1 antibody and $\beta$ endorphin antibody were purchased from Shanghai Zhuocai Biotech Co., Ltd.

\section{Animal modeling}

The BPI animal model was established according to previously reported (5). Rats received food and water deprivation for $12 \mathrm{~h}$ before study. They were weighed and then intraperitoneally anesthetized with $1 \%$ pentobarbital sodium at $40 \mathrm{mg} / \mathrm{kg}$. Then, rats were placed in a supine position and the limbs were fixed on a table. The chest and abdomen were exposed, and surgery was done at the right side. The skin from the middle neck to the area $1-2 \mathrm{~cm}$ besides the sternal stem was sterilized and a horizontal $1.5-\mathrm{cm}$ incision was made at $2 \mathrm{~mm}$ below the clavicle. The skin and subcutaneous tissues were separated and the pectoralis major was exposed. The pectoralis major was cut along the direction of muscular fibers to expose the brachial plexus. The cephalic vein was remained intact. The subclavian vein was positioned and then the C5-T1 nerve roots were separated and retracted until the ganglions separated from the intervertebral foramen. The ganglions were then placed on the surface of anterior cervical muscle group. After complete hemostasis, penicillin was locally administered, followed by wound closure.

\section{Grouping}

A total of 72 SD rats were randomly assigned into 3 groups ( $n=24$ per group). Blank control group: animals had no any treatment and were routinely housed. BPI group: BPI was induced, and animals had no other treatment and were routinely housed. Naprapathy group: BPI was induced as previously reported, naprapathy was performed at the C5T1 Jiaji Point (the site the brachial plexus nerves arise from) for 15 min daily on the 4th day after modeling and animals 
were routinely housed. The treatment was lasted for 28 days altogether; 7, 14 and 21 days after naprapathy, 6 rats in each group were subjected to behavioral examinations.

\section{Naprapathy therapy}

\section{Procedures for naprapathy}

The physicians received naprapathy training before study. The frequency and pressure during naprapathy were controlled and standardized. Rats were placed in a fixation box and the end of the forefinger ( $5 \mathrm{~mm}$ in diameter) was used for naprapathy. The pressure was $2 \mathrm{~N}$ and the frequency was 60 per minute.

\section{Site position}

Naprapathy was performed at the C5-T1 Jiaji Point (the site the brachial plexus nerves arise from).

\section{Protocol}

At 4 days after model establishment, rats were fixed on a table in a supine position, and a spring dynamometer was suspended above the animal to control the pressure. A timemeter was used to control the frequency of naprapathy. Naprapathy was performed for 15 min once daily.

\section{Behavioral tests}

\section{Mechanical withdrawal threshold (MWT)}

Rats were housed for 1 week. The MWT was determined 1 day before surgery, 3 days after surgery and 7, 14, 21 and 28 days after naprapathy. In brief, rats were placed on a stainless-steel mesh mat in a transparent cage and allowed to move freely. Animals were allowed to accommodate to the environment for $10 \mathrm{~min}$, and pain measurement was done with the Electric Von Frey detector when the exploration activity disappeared. The probe was used to slowly stimulate the hind paw along the direction vertical to the paw. The pressure was recorded as the MWT when the paw withdrew. Measurement was done 5 times with an interval of $5 \mathrm{~min}$ between measurements. The maximal or minimal values were not used for analysis and the mean was calculated.

\section{Allodynia test}

Rats were housed for 1 week. The response to innocuous cold stimulus was determined 1 day before surgery, 3 days after surgery and 7, 14, 21 and 28 days after naprapathy. In brief, rats were placed on a cold plate at $4{ }^{\circ} \mathrm{C}$ in a transparent cage and allowed to move freely. Animals were allowed to accommodate to the environment for $3 \mathrm{~min}$, and the number of paw lifting was recording within $15 \mathrm{~min}$. The paw lifting due to activity or body movement was not counted (6).

\section{Detection of $\beta$-endorphin and GABA in the thalamus by immunofluorescence staining}

Animals were sacrificed and the brain was collected. The brain tissues were embedded in paraffin and then sectioned. The brain sections were subsequently deparaffinized in xylene and ethanol. Antigen retrieval was done in citric acid solution, and the sections were rinsed in PBS (pH7.4) thrice (5 min for each). Then, sections were blocked with 3\% BSA at room temperature for $30 \mathrm{~min}$. The primary antibody solution was added, followed by incubation in a humidified box at $4{ }^{\circ} \mathrm{C}$ over night. The second antibody solution was added, followed by incubation at room temperature for 50 min in dark. After washing in PBS (PH7.4) thrice (5 min for each), sections were mounted. Sections were observed under an inverted fluorescence microscope and images were captured at ultraviolet excitation wavelength of $330-380 \mathrm{~nm}$, ultraviolet emission wavelength of $420 \mathrm{~nm}$, FITC excitation wavelength of 465-495 nm, FITC emission wavelength of $515-555 \mathrm{~nm}, \mathrm{CY} 3$ excitation wavelength of 510-560 nm and CY3 emission wavelength of $590 \mathrm{~nm}$. DAPI positive nucleus was blue. Image analysis was done with Image-Pro Plus 6.0. The mean positive immunoreactivity was determined in positive cells after standardization of grayscale and brightness.

\section{Statistical analysis}

Statistical analysis was performed with SPSS version 21.0. The normal distribution and variance homogeneity were tested before statistical analysis. Data with normal distribution and variance homogeneity were compared with analysis of variance among groups, followed by LSD test between two groups; Games-Howell test was employed for comparisons if heterogeneity of variance was noted. Data with abnormal distribution and/or heterogeneity of variance, non-parametric Friedman rank sum test was employed. A value of $\mathrm{P}<0.05$ was considered statistically significant.

\section{Results}

\section{Behavioral tests}

After gaining consciousness, rats developed evident 

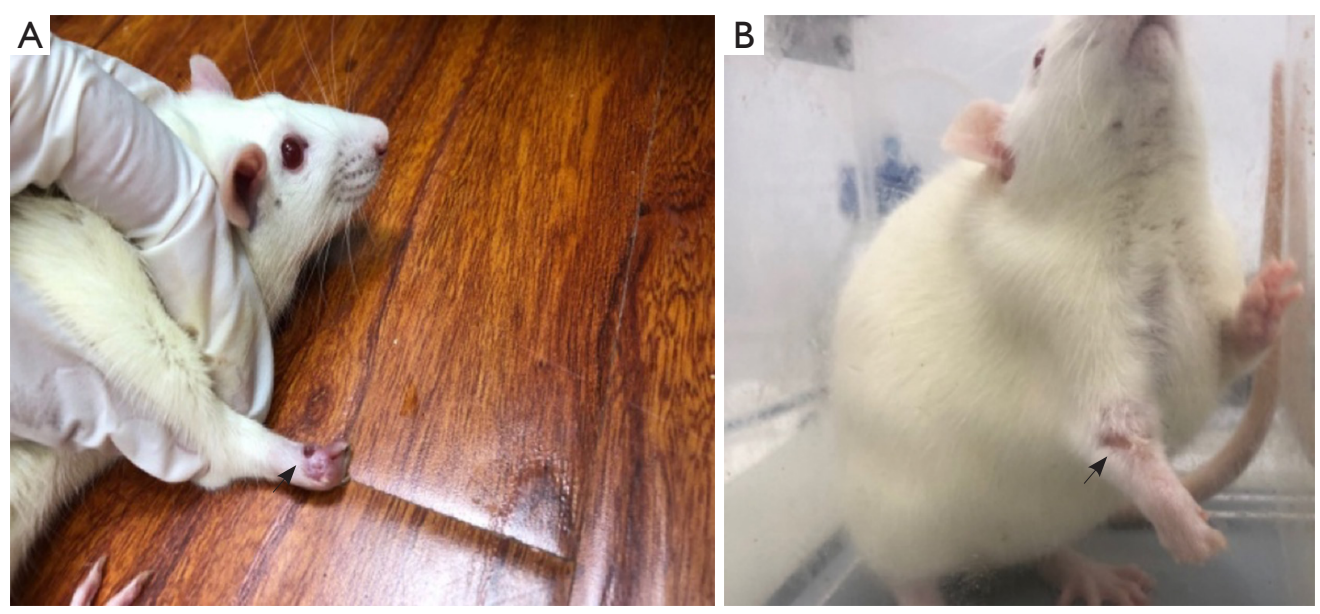

Figure 1 Deficiency of nails (A) and limb contracture (B). Arrow: right limb contracture with concomitant deficiency of nails.

Table 1 MWT of rats in different groups at different time points after modeling $(\bar{x} \pm S D)$

\begin{tabular}{lccc}
\hline Time points & Blank control group & BPI group & Naprapathy group \\
\hline Before modeling & $29.93 \pm 3.33$ & $29.22 \pm 3.42$ & $29.09 \pm 2.86$ \\
After modeling & $30.72 \pm 2.50$ & $23.65 \pm 2.67^{\star \star}$ & $22.81 \pm 3.27^{\star \star}$ \\
After 7-day treatment & $33.41 \pm 3.05$ & $23.36 \pm 2.87^{\star *}$ & $24.09 \pm 2.76^{\star \star}$ \\
After 14-day treatment & $34.06 \pm 3.80$ & $24.66 \pm 4.35^{\star *}$ & $30.28 \pm 5.43^{\star \# \#}$ \\
After 21-day treatment & $38.03 \pm 5.01$ & $27.77 \pm 4.28^{\star *}$ & $34.68 \pm 5.35^{\# \#}$ \\
After 28-day treatment & $39.04 \pm 3.36$ & $27.87 \pm 4.90^{\star *}$ & $38.97 \pm 4.50^{\# \#}$ \\
\hline
\end{tabular}

*, $\mathrm{P}<0.05$ vs. blank control group; ${ }^{\star \star}, \mathrm{P}<0.01$ vs. blank control group; ${ }^{\# \#, ~} \mathrm{P}<0.01$ vs. BPI group. MWT, mechanical withdrawal threshold; $\mathrm{BPI}$, brachial plexus injury.

lameness or dragging leg and the affected limb presented swelling. Three days after modeling, the right limb became evidently contractural, the food intake reduced, and the hairy luster decreased. These rats were susceptible to irritation, the fighting with each other increased over time, and the muscle of affected limb became contractural. These manifestations were not improved 31 days after modeling, and some animals developed deficiency of nails, which was more evident in the BPI group (Figure 1A,B). After naprapathy, the food intake increased, the fighting with each other reduced, and the deficiency of nails was also improved although other behavioral manifestations remained.

\section{$M W T$}

In the blank control group, BPI group and naprapathy group, the MWT was $29.93 \pm 3.33,29.22 \pm 3.42$ and $29.09 \pm 2.86$, respectively, before modeling showing no significant difference among groups at baseline. After modeling, the MWT in BPI group and naprapathy group was significantly different from that in the blank control groups $(\mathrm{P}<0.01) ; 14$ days after naprapathy, significant difference was noted in the MWT between naprapathy group and blank control group $(\mathrm{P}<0.05)$, between BPI group and blank control group $(\mathrm{P}<0.01)$ and between BPI group and naprapathy group $(\mathrm{P}<0.01) ; 21$ and 28 days after naprapathy, the MWT in the BPI group was significantly different from that in the blank control group and naprapathy group $(\mathrm{P}<0.01)$, but there was no significant difference between the naprapathy group and the blank control group $(\mathrm{P}>0.05)$ (Table 1).

\section{Number of paw lifting}

The number of paw lifting was $14.13 \pm 4.61,11.75 \pm 3.43$ and $13.79 \pm 4.84$ in the blank control group, BPI group and the naprapathy group, respectively, before modeling, showing 
Table 2 Number of paw lifting in rats in different groups at different time points $(\bar{x} \pm S D)$

\begin{tabular}{lccc}
\hline Time points & Blank control group & BPI group & Naprapathy group \\
\hline Before modeling & $14.13 \pm 4.61$ & $11.75 \pm 3.43$ & $13.79 \pm 4.84$ \\
After modeling & $12.63 \pm 5.11$ & $9.71 \pm 5.07^{\star}$ & $10.38 \pm 5.15$ \\
After 7-day treatment & $13.00 \pm 5.05$ & $10.63 \pm 6.26$ & $13.29 \pm 9.26$ \\
After 14-day treatment & $12.61 \pm 5.67$ & $10.83 \pm 7.68$ & $13.94 \pm 9.71$ \\
After 21-day treatment & $13.08 \pm 8.81$ & $10.00 \pm 6.00$ & $13.58 \pm 6.29$ \\
After 28-day treatment & $10.17 \pm 1.47$ & $11.17 \pm 12.29$ & $12.50 \pm 12.63$ \\
\hline
\end{tabular}

${ }^{*}, \mathrm{P}<0.05$ vs. blank control group.
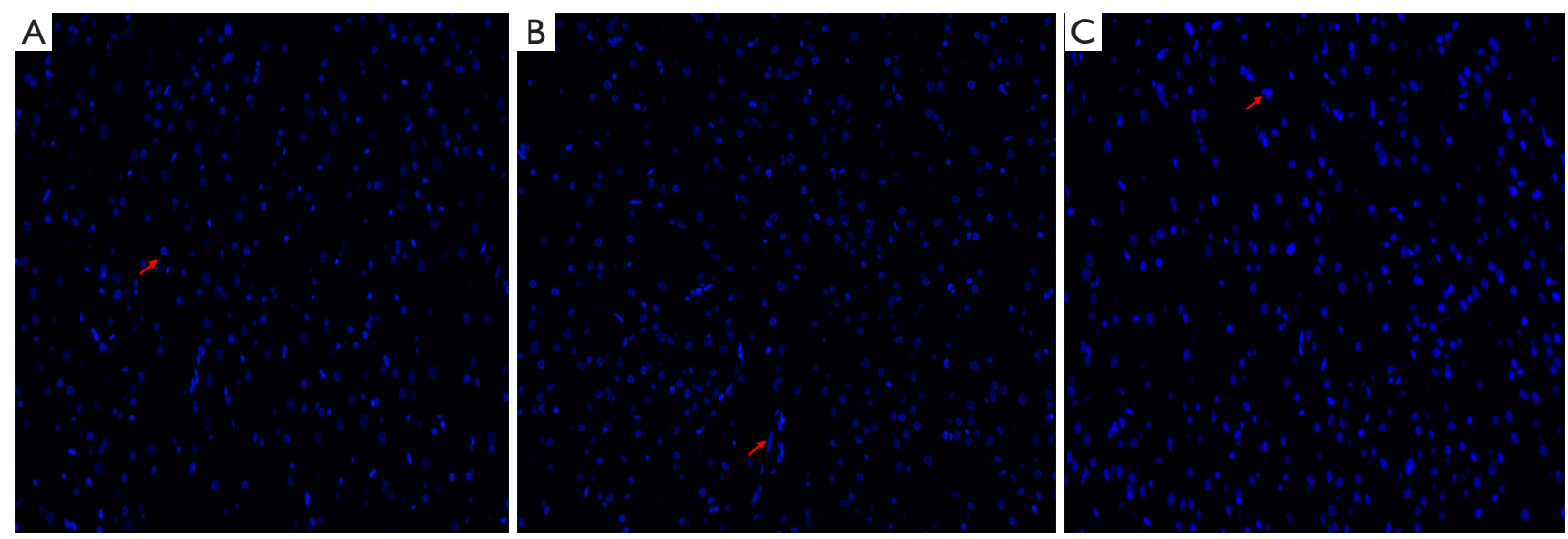

Figure $2 \beta$-endorphin expression in the thalamus after 28-day naprapathy (immunofluorescence staining; 200x). (A) Blank control group; (B) BPI group; (C) naprapathy group. Note: blue, $\beta$-endorphin positive cells. Arrows indicate the positive cell. BPI, brachial plexus injury.

Table $3 \beta$-endorphin expression in the thalamus of rats in different groups at different time points $(\bar{x} \pm S D)$

\begin{tabular}{lccc}
\hline Time points & Blank control group & BPI group & Naprapathy group \\
\hline After 7-day treatment & $0.0769 \pm 0.0116$ & $0.0650 \pm 0.0159$ & $0.0793 \pm 0.0282$ \\
After 14-day treatment & $0.0640 \pm 0.0121$ & $0.0699 \pm 0.0084$ & $0.0953 \pm 0.0482$ \\
After 21-day treatment & $0.0690 \pm 0.0097$ & $0.0657 \pm 0.0093$ & $0.0946 \pm 0.0082$ \\
After 28-day treatment & $0.0647 \pm 0.0328$ & $0.0648 \pm 0.0024$ & $0.1060 \pm 0.0070^{* \#}$ \\
\hline
\end{tabular}

*, $\mathrm{P}<0.05$ vs. blank control group; ${ }^{\text {, }} \mathrm{P}<0.05$ vs. BPI group. BPI, brachial plexus injury.

no significant difference among groups at baseline. Three days after modeling, significant difference in the number of paw lifting was only observed between the BPI group and the blank control group $(\mathrm{P}<0.05)$ (Table 2$)$.

\section{$\beta$-endorphin expression in the thalamus}

Seven, 14 and 21 days after naprapathy, there was no significant difference in the $\beta$-endorphin expression among the blank control group, BPI group and naprapathy $(\mathrm{P}>0.05)$. After 28-day naprapathy (Figure 2), the $\beta$-endorphin expression in the blank control group was significantly different from that in the naprapathy group $(\mathrm{P}<0.05)$, and significant difference was also noted between the BPI group and the naprapathy group $(\mathrm{P}<0.05)$ (Table 3). 

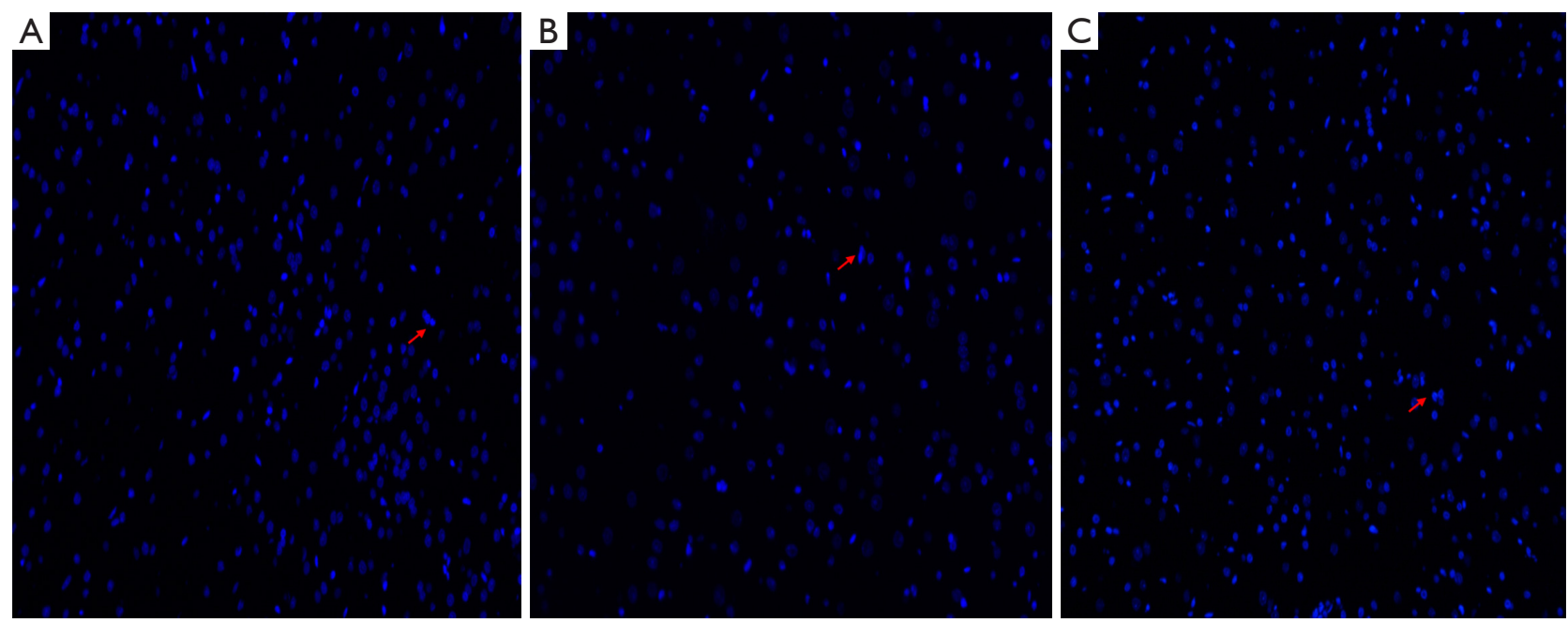

Figure 3 GABA content of the thalamus after 28-day naprapathy (immunofluorescence staining; 200x). (A) Blank control group; (B) BPI group; (C) naprapathy group. Note: blue, GABA positive cells. Arrows indicate the positive cell. GABA, $\gamma$-aminobutyric acid; BPI, brachial plexus injury.

Table 4 GABA content of the thalamus $(\bar{x} \pm S D)$

\begin{tabular}{lccc}
\hline Time points & Blank control group & BPI group & Naprapathy group \\
\hline After 7-day treatment & $0.0730 \pm 0.0099$ & $0.0569 \pm 0.0246$ & $0.0804 \pm 0.0159$ \\
After 14-day treatment & $0.0730 \pm 0.0134$ & $0.0532 \pm 0.0179$ & $0.0857 \pm 0.0132^{\#}$ \\
After 21-day treatment & $0.0720 \pm 0.0165$ & $0.0697 \pm 0.0240$ & $0.0880 \pm 0.0311$ \\
After 28-day treatment & $0.0691 \pm 0.0127$ & $0.0584 \pm 0.0144$ & $0.0919 \pm 0.0186^{\#}$ \\
\hline
\end{tabular}

\#, P<0.05 vs. BPI group. GABA, $\gamma$-aminobutyric acid; BPI, brachial plexus injury.

\section{GABA content of the thalamus}

Seven days after naprapathy, the GABA content of the thalamus was similar among the blank control group, BPI group and naprapathy group $(\mathrm{P}>0.05) ; 14$ days after naprapathy, significant difference in the GABA content was found between the BPI group and the naprapathy group $(\mathrm{P}<0.05) ; 21$ days after naprapathy, the GABA content of the thalamus was comparable among groups $(\mathrm{P}>0.05)$; 21 days after naprapathy, there was significant difference in the GABA content between the BPI group and the naprapathy group $(\mathrm{P}<0.05)$ (Figure 3, Table 4).

\section{Discussion}

In the present study, the MWT, thalamic beta-endorphin expression and thalamic GABA content were detected in BPI rats after naprapathy, and our results indicated that naprapathy could improve the NP after BPI. BPI can be divided into pre- and post-ganglionic one. The post-ganglionic injury is also known as brachial plexus root avulsion and is the most serious type of BPI. The NP secondary to BPI may occur immediately or several months after injury, and the severity of NP will increase over time (7). NP is also a type of chronic pain that results from the direct injury to or the dysfunction of the peripheral or central nervous system $(8,9)$. The underlying mechanism of NP may have involvement of damage to the afferent transmission system. Depending on the type of affected afferent nerves, sensory dysfunction may be present. This pain syndrome caused by the damage to or the disease of the central or peripheral nervous system is often characterized by spontaneous pain, hyperalgia and hyperalgesia. Our results showed that the BPI might not cause cold hyperalgesia in rats, which may be related to the BPI induced burning like pain in patients. 
After nerve injury, the noxious stimulation may induce the release of EAAs in the afferent nerve endings of the spinal cord, which activates the NMDA receptor, causes excessive $\mathrm{Ca} 2+$ influx, activates protein kinases, and leads to the production of factors involved in the transmission of noxious information media, which increase the excitability of numerous pathways in the spinal cord and other central nervous system and result in the central sensitization of spinal dorsal horn neurons, causing mechanical hyperalgesia (10). Our results showed the MWT of rats with BPI decreased significantly. Seven days after naprapathy, the MWT remained unchanged, but it increased significantly 14 days after naprapathy, and prolonged naprapathy (21 and 28 days) further increased the MWT.

Endorphin is an endogenous morphine-like biochemically synthesized hormone and possesses morphine-like activity. It can exert analgesic effect and regulatory effects on the body temperature, cardiovascular function, and respiratory function. The noxious stimuli may enhance the release of $\beta$-endorphin, which then relax the human body and the pain is then relieved (11-13). $\beta$-endorphin has been recognized as one of the neurotransmitters associated with analgesia, and thus $\beta$-endorphin expression was detected in the present study. Our results revealed that naprapathy increased the $\beta$-endorphin expression in the thalamus.

$\gamma$-aminobutyric acid (GABA) is one of four major types of neurotransmitters associated with anxiety. In the endogenous analgesic system, GABAergic neurons participate in pain modulation, and the noxious stimuli may increase the production of endogenous GABA, exerting analgesic effect $(14,15)$. According to the shared mechanism between pain and depression, GABA might participate in the analgesic effect of naprapathy, and thus GABA content was detected in the thalamus. Our results indicated naprapathy increased the GABA content of the thalamus.

In summary, naprapathy can improve the MWT of rats with BPI, effectively alleviate NP in these rats, and then improve their behaviors. Naprapathy can also increase the $\beta$-endorphin expression and GABA content in the brain of rats with BPI, which may be one of mechanisms underlying the analgesic effect of naprapathy. Naprapathy may exert analgesic effect to a certain extent and thus may be employed as an alternative treatment of NP secondary to BPI.

\section{Acknowledgments}

Funding: This work was supported by the Fund of Shanghai University of Traditional Chinese Medicine (No.
2019LK080); The Three-year Development Project for Traditional Chinese Medicine of Shanghai [No. ZY(20182020)-CCCX-2001-05].

\section{Footnote}

Conflicts of Interest: All authors have completed the ICMJE uniform disclosure form (available at http://dx.doi. org/10.21037/apm.2020.04.16). The authors have no conflicts of interest to declare.

Ethical Statement: The authors are accountable for all aspects of the work in ensuring that questions related to the accuracy or integrity of any part of the work are appropriately investigated and resolved. The treatment of animals conformed to the ethical criteria (SZY201805012).

Open Access Statement: This is an Open Access article distributed in accordance with the Creative Commons Attribution-NonCommercial-NoDerivs 4.0 International License (CC BY-NC-ND 4.0), which permits the noncommercial replication and distribution of the article with the strict proviso that no changes or edits are made and the original work is properly cited (including links to both the formal publication through the relevant DOI and the license). See: https://creativecommons.org/licenses/by-nc-nd/4.0/.

\section{References}

1. Lao J, Gao K. Functional Anatomy of Brachial Plexus. 2019:289-315.

2. Rasulic L, Savic A, Zivkovic B, et al. Outcome after brachial plexus injury surgery and impact on quality of life. Acta Neurochir (Wien) 2017;159:1257-64.

3. Edwards RR, Dworkin RH, Sullivan MD, et al. The Role of Psychosocial Processes in the Development and Maintenance of Chronic Pain. J Pain 2016;17:T70-92.

4. Boogaard S, Heymans MW, de Vet HC, et al. Predictors of Persistent Neuropathic Pain--A Systematic Review. Pain Physician 2015;18:433-57.

5. Wang L, Yuzhou L, Yingjie Z, et al. A new rat model of neuropathic pain: complete brachial plexus avulsion. Neurosci Lett 2015;589:52-6.

6. Bennett GJ, Xie YK. A peripheral mononeuropathy in rat that produces disorders of pain sensation like those seen in man. Pain 1988;33:87-107.

7. Lovaglio AC, Socolovsky M, Di Masi G, et al. Treatment of neuropathic pain after peripheral nerve and brachial 
plexus traumatic injury. Neurol India 2019;67:S32-7.

8. Widerstrom-Noga E. Neuropathic Pain and Spinal Cord Injury: Phenotypes and Pharmacological Management. Drugs 2017;77:967-84.

9. Zilliox LA. Neuropathic Pain. Continuum (Minneap Minn) 2017;23:512-32.

10. Wang C, Li Y, Wang H, et al. Inhibition of DOR prevents remifentanil induced postoperative hyperalgesia through regulating the trafficking and function of spinal NMDA receptors in vivo and in vitro. Brain Res Bull 2015;110:30-9.

11. He XF, Wei JJ, Shou SY, et al. Effects of electroacupuncture at 2 and $100 \mathrm{~Hz}$ on rat type 2 diabetic neuropathic pain and hyperalgesia-related protein expression in the dorsal root ganglion. J Zhejiang Univ Sci B 2017;18:239-48.

12. Mazzardo-Martins L, Salm DC, Winkelmann-Duarte EC,

Cite this article as: Xiao B, Ma A, Li Z, Zhang S, Xu X, Zhou J, Li W, Zhang J, Yao F. Naprapathy attenuates neuropathic pain after brachial plexus injury. Ann Palliat Med 2020;9(3):766-773. doi: 10.21037/apm.2020.04.16 et al. Electroacupuncture induces antihyperalgesic effect through endothelin-B receptor in the chronic phase of a mouse model of complex regional pain syndrome type I. Pflugers Arch 2018;470:1815-27.

13. Tobaldini G, Sardi NF, Guilhen VA, et al. Pain Inhibits Pain: an Ascending-Descending Pain Modulation Pathway Linking Mesolimbic and Classical Descending Mechanisms. Mol Neurobiol 2019;56:1000-13.

14. Ochoa-de la Paz L, Zenteno E, Gulias-Canizo R, et al. Taurine and GABA neurotransmitter receptors, a relationship with therapeutic potential? Expert Rev Neurother 2019;19:289-91.

15. Rytova V, Ganella DE, Hawkes D, et al. Chronic activation of the relaxin-3 receptor on GABA neurons in rat ventral hippocampus promotes anxiety and social avoidance. Hippocampus 2019;29:905-20. 